Supplemental Information for:

\title{
One-Step Multi-Functionalization of Random Copolymers via Self-Assembly
}

\author{
Joel M. Pollino, Ludger P. Stubbs, and Marcus Weck \\ Georgia Institute of Technology; School of Chemistry and Biochemistry; Atlanta, GA 30332-0400
}

General Methods. $\mathrm{Ru}(=\mathrm{Ph}) \mathrm{Cl}_{2}\left(\mathrm{PCy}_{3}\right)_{2}$ was purchased from Strem. All monomers and homo-polymers were prepared as previously reported. ${ }^{1} \mathrm{CDCl}_{3}$ was distilled from calcium hydride and degassed prior to use. NMR spectra were taken using a $400 \mathrm{MHz}$ Bruker AMX 400 spectrometer. All spectra are referenced to residual proton solvent. FTIR data was obtained using a Shimadzu FTIR-8400S infrared spectrophotometer with samples prepared via flash evaporation of polymer solutions $\left(\mathrm{CH}_{2} \mathrm{Cl}_{2}\right)$ onto salt plates. UVvis data was collected using a Shimadzu UV-2401PC UV-VIS recording spectrophotometer. Gel permeation chromatography (GPC) analyses for $\mathbf{3 a}$ and $\mathbf{4 a}$ were carried out using a Waters 1525 binary pump coupled to a Waters 2414 refractive index detector with $\mathrm{CH}_{2} \mathrm{Cl}_{2}$ as an eluant on American Polymer Standards 10 _ particle size, linear mixed bed packing columns (2x). GPC analysis for $\mathbf{5 a}, \mathbf{6 a}, \mathbf{7 a}$, were carried out using a waters 510 binary pump coupled to a Waters 410 differential refractometer with THF as an eluant on an American Polymer Standards column set (100 $\AA, 1000 \AA, 100,000 \AA$, linear mixed bed). All GPCs were calibrated using polystyrene standards. Differential scanning calorimetry (DSC) was performed under nitrogen using a Perkin-Elmer DSC 7 equipped with an Intracooler II cooling device. The temperature program provided heating and cooling cycles between 5 and $200{ }^{\circ} \mathrm{C}$ at $40{ }^{\circ} \mathrm{C} / \mathrm{min}$. Thermal gravimetric analysis (TGA) was performed under nitrogen using a Perkin-Elmer TGA 7 and all samples were heated from $50-500{ }^{\circ} \mathrm{C}$.

Copolymerization (4a-6a). Monomers 1 and 2 were weighed, dissolved in degassed, anhydrous $\mathrm{CDCl}_{3}(1 \mathrm{~mL}$ per $100 \mathrm{mg}$ ), and stirred under an atmosphere of argon. A stock solution of $\mathrm{Ru}(=\mathrm{Ph}) \mathrm{Cl}_{2}\left(\mathrm{PCy}_{3}\right)_{2}$ was prepared and the desired amount added to the monomer feedstock in one portion. Upon complete polymerization (determined in situ via ${ }^{1} \mathrm{H}$ NMR spectroscopy), a drop of ethyl vinyl ether was added to terminate the polymerization. The final polymers were isolated as dark green solids by precipitation from hexanes followed by prolonged drying on high vacuum (isolated yields: 90$95 \%)$. 
${ }^{1} \mathrm{H}$ NMR $\left(\mathrm{CD}_{2} \mathrm{Cl}_{2}\right): \square=7.93(\mathrm{br} \mathrm{s}, 2 \mathrm{H}, \mathrm{CONH}), 7.83-7.81(\mathrm{~m}, 4 \mathrm{H}, \mathrm{SPh}), 7.48\left(\mathrm{~m}, 2 \mathrm{H}, \mathrm{Pyr}_{\square}\right), 7.38-7.36$ (m, 6H, SPh), $6.57(\mathrm{~s}, 2 \mathrm{H}, \mathrm{ArH}), 5.42-5.17(\mathrm{br} \mathrm{m}, 4 \mathrm{H}, \mathrm{CH}=\mathrm{CH}), 4.55\left(\mathrm{~s}, 4 \mathrm{H}, \mathrm{CH}_{2} \mathrm{~S}\right), 4.07-3.94$ (br m, $6 \mathrm{H}, \mathrm{CH}_{2} \mathrm{O}$ ), 3.84 (br t, $\mathrm{J}=6.4 \mathrm{~Hz}, 2 \mathrm{H}, \mathrm{CH}_{2} \mathrm{O}$ ), 3.16-2.40 (br m, 6H), 2.32 (br m, 4H, $\mathrm{CH}_{2} \mathrm{CH}_{3}$ ), 2.121.50 (br m, 16H), 1.46-1.07 (br m, 28H), 1.14 (br t, J = 7.4 Hz, 6H, $\mathrm{CH}_{2} \mathrm{CH}_{3}$ ).

${ }^{13} \mathrm{C}$ NMR $\left(\mathrm{CD}_{2} \mathrm{Cl}_{2}\right): \square=176.2,173.0,169.3,157.7,152.0,151.4,150.7,134.2,133.1,131.9,130.3$, $130.1,109.4,96.1,69.0,68.6,64.9,52.2,50.8,48.3,42.6,41.6,36.8,35.2,35.1,32.2,30.1,29.9-$ 29.7, 29.5, 29.3, 26.5, 26.4, 25.8, 21.0, 9.6.

FTIR (NaCl): $\mathrm{cm}^{-1}=3277,3054,2927,2853,1727,1698,1615,1582,1537,1505,1438,1392,1354$, $1316,1266,1210,1162,1043,1024,967,890,847,742,687$.

UVVIS $\left(\mathrm{CH}_{2} \mathrm{Cl}_{2}\right): \square_{\max }(\mathrm{nm})=278(\mathrm{sh}), 304(\mathrm{sh}), 335$.

Directed Self-Assembly: Pyridine to Pd" Pincer Complexes (4b-6b). Copolymers 4a-6a were dissolved in $\mathrm{CD}_{2} \mathrm{Cl}_{2}(0.7 \mathrm{~mL}$ per $50 \mathrm{mg})$. To these solutions, one stoichiometric equivalent (based on the number of pincer complexes) of a pyridine stock solution (in $\mathrm{CD}_{2} \mathrm{Cl}_{2}$ ) was added. Subsequently, one equivalent of $\mathrm{AgBF}_{4}$ (stock solution in nitromethane) was introduced to the mixture and the color changed from dark to pale green. Upon complete coordination, crude polymers $4 \mathbf{b}-\mathbf{6 b}$ were obtained by filtration over Celite $\AA$, removal of the solvent, and drying on high vacuum. To remove excess $\mathrm{AgCl}$ salts, the polymers were subjected to repeated (3X) redissolution in $\mathrm{CH}_{2} \mathrm{Cl}_{2}$ followed by filtration over Celite ${ }^{\circledR}$, removal of the solvent, and extensive drying on high vacuum to provide pure $\mathbf{4 b - 6 b}$.

${ }^{1} \mathrm{H}$ NMR $\left(\mathrm{CD}_{2} \mathrm{Cl}_{2}\right): \square=8.04\left(\mathrm{~s}, 2 \mathrm{H}, \mathrm{Pyr}_{\square} \mathrm{H}\right), 7.93(\mathrm{br} \mathrm{s}, 2 \mathrm{H}, \mathrm{CONH}), 7.76\left(\mathrm{~m}, 1 \mathrm{H}, \mathrm{Pyr}_{\mathrm{d}} \mathrm{H}\right), 7.63(\mathrm{~m}, 4 \mathrm{H}$, SPh), 7.49-7.37 (br m, 8H, SPh \& Pyr $\left._{\square}\right), 7.27$ (m, 2H, Pyr $\left.r_{\square}\right), 6.68$ (s, 2H, ArH), 5.42- 5.17 (br m, 4H, $\mathrm{CH}=\mathrm{CH}$ ), 4.74 (br s, $4 \mathrm{H}, \mathrm{CH}_{2} \mathrm{~S}$ ), 4.07-3.94 (br m, 6H, $\mathrm{CH}_{2} \mathrm{O}$ ), 3.84 (br t, J = 6.4 Hz, 2H, $\mathrm{CH}_{2} \mathrm{O}$ ), 3.16- 
2.40 (br m, 6H), 2.32 (br m, 4H, $\left.\mathrm{CH}_{2} \mathrm{CH}_{3}\right), 2.12-1.50$ (br m, 16H), 1.46-1.07 (br m, 28H), 1.14 (br t, $J=$ $\left.7.4 \mathrm{~Hz}, 6 \mathrm{H}, \mathrm{CH}_{2} \mathrm{CH}_{3}\right)$.

${ }^{13} \mathrm{C}$ NMR $\left(\mathrm{CD}_{2} \mathrm{Cl}_{2}\right): \square=176.2,173.0,169.3,158.4,151.5,150.7,147.3,139.7,134.2,132.0,131.5$, $131.1,130.8,126.7,110.1,96.1,69.0,68.6,64.9,52.0,50.8,48.3,42.6,41.6,36.8,35.2,35.1,32.2$, $30.1,29.9-29.8,29.5,29.3,26.5,26.4,25.8,21.0,9.6$.

FTIR $(\mathrm{NaCl}): \mathrm{cm}^{-1}=3277,3054,2927,2854,1725,1694,1615,1580,1547,1512,1437,1397,1316$, 1267, 1208, 1161, 1062, 1029, 970, 893, 844, 749, 693.

UVVIS $\left(\mathrm{CH}_{2} \mathrm{Cl}_{2}\right): \square_{\max }(\mathrm{nm})=278(\mathrm{sh}), 315(\mathrm{sh})$

\section{Directed Self-Assembly: Butylthymine to Diaminopyridine Moieties (4c-6c). Copolymers 4a-6a} were dissolved in $\mathrm{CD}_{2} \mathrm{Cl}_{2}(0.7 \mathrm{~mL}$ per $50 \mathrm{mg})$. To these solutions, 1.5 stoichiometric equivalents of $\mathrm{N}$ butylthymine (based on the number of diaminopyridine moieties: this is the point where the chemical shift of the amide protons no longer changes) were added to the solution and stirred for $5 \mathrm{~min}$. The final polymers were obtained by rota-evaporation of the solvent followed by drying on high vacuum.

${ }^{1} \mathrm{H}$ NMR $\left(\mathrm{CD}_{2} \mathrm{Cl}_{2}\right): \square=10.53(\mathrm{br} \mathrm{s}, 1 \mathrm{H}, \mathrm{NH}), 9.66$ (br s, 2H, CONH), 7.83-7.81 (m, 4H, SPh), $7.61(\mathrm{~m}$, $\left.2 \mathrm{H}, \mathrm{Pyr}_{\square}\right), 7.38-7.36(\mathrm{~m}, 6 \mathrm{H}, \mathrm{SPh}), 7.06\left(\mathrm{~m}, 1 \mathrm{H}, \mathrm{CH}=\mathrm{CCH}_{3}\right), 6.57(\mathrm{~s}, 2 \mathrm{H}, \mathrm{ArH}), 5.42-5.17$ (br m, 4H, $\mathrm{CH}=\mathrm{CH}$ ), 4.55 (br s, 4H, $\left.\mathrm{CH}_{2} \mathrm{~S}\right), 4.07-3.94$ (br m, 6H, $\left.\mathrm{CH}_{2} \mathrm{O}\right), 3.84$ (br t, J = 6.4 Hz, 2H, $\mathrm{CH}_{2} \mathrm{O}$ ), $3.70(\mathrm{t}$, $\left.J=7.2 \mathrm{~Hz}, 2 \mathrm{H}, \mathrm{CH}_{2} \mathrm{~N}\right), 3.16-2.40$ (br m, 10H), 2.12-1.50 (br m, 21H), 1.46-1.07 (br m, 30H), 1.14 (br t, $\left.J=7.4 \mathrm{~Hz}, 6 \mathrm{H}, \mathrm{CH}_{2} \mathrm{CH}_{3}\right), 0.95\left(\mathrm{t}, J=7.4 \mathrm{~Hz}, 3 \mathrm{H}, \mathrm{CH}_{2} \mathrm{CH}_{3}\right)$.

${ }^{13} \mathrm{C}$ NMR $\left(\mathrm{CD}_{2} \mathrm{Cl}_{2}\right): \square=176.2,174.2,169.3,165.9,157.7,152.6,152.4,150.7,141.5,133.1,131.9$, $130.3,130.1,111.0,109.4,96.1,96.8,69.0,68.6,64.9,52.4,50.8,50.1,48.7,48.3,42.6,41.6,36.8$, $35.2,35.1,32.2,31.6,30.5,30.2-29.8,29.7,29.5,29.3,26.5,26.4,20.2,14.0,12.5,9.08$. 
FTIR $(\mathrm{NaCl}): \mathrm{cm}^{-1}=3269,3214,3162,3053,2930,2857,1683,1578,1546,1436,1355,1315,1274$, $1217,1165,1052,1012,965,933,895,846,747,690$.

UVVIS $\left(\mathrm{CH}_{2} \mathrm{Cl}_{2}\right): \square_{\max }(\mathrm{nm})=271(\mathrm{sh}), 304(\mathrm{sh}), 335$.

Multi-Step Self-Assembly: Starting with Metal-Coordination (4d- 6d). Copolymers $4 b-6 b$ were dissolved in $\mathrm{CD}_{2} \mathrm{Cl}_{2}(0.7 \mathrm{~mL}$ per $50 \mathrm{mg})$. To these solutions, 1.5 stoichiometric equivalents of $\mathrm{N}$ butylthymine (based on the number of diaminopyridine moieties) was added to the solution and stirred for $5 \mathrm{~min}$. The final polymers were obtained by rota-evaporation of the solvent followed by drying on high vacuum.

${ }^{1} \mathrm{H}$ NMR $\left(\mathrm{CD}_{2} \mathrm{Cl}_{2}\right): \square=10.53(\mathrm{br} \mathrm{s}, 1 \mathrm{H}, \mathrm{NH}), 9.66(\mathrm{br} \mathrm{s}, 2 \mathrm{H}, \mathrm{CONH}), 8.04\left(\mathrm{~s}, 2 \mathrm{H}, \mathrm{Pyr}_{\square} \mathrm{H}\right), 7.76(\mathrm{~m}, 1 \mathrm{H}$, $\left.\mathrm{Pyr}_{\mathbb{0}} \mathrm{H}\right), 7.63\left(\mathrm{~m}, 6 \mathrm{H}, \mathrm{SPh} \& \mathrm{Pyr}_{\square}\right), 7.49-7.37$ (br m, 6H, SPh), $7.27\left(\mathrm{~m}, 2 \mathrm{H}, \mathrm{Pyr}_{\square}\right), 7.06(\mathrm{~m}, 1 \mathrm{H}$, $\left.\mathrm{CH}=\mathrm{CCH}_{3}\right), 6.68(\mathrm{~s}, 2 \mathrm{H}, \mathrm{ArH}), 5.42-5.17(\mathrm{br} \mathrm{m}, 4 \mathrm{H}, \mathrm{CH}=\mathrm{CH}), 4.74\left(\mathrm{br} \mathrm{s}, 4 \mathrm{H}, \mathrm{CH}_{2} \mathrm{~S}\right), 4.07-3.94(\mathrm{br} \mathrm{m}$, $6 \mathrm{H}, \mathrm{CH}_{2} \mathrm{O}$ ), $3.84\left(\mathrm{brt}, J=6.4 \mathrm{~Hz}, 2 \mathrm{H}, \mathrm{CH}_{2} \mathrm{O}\right.$ ), $3.70\left(\mathrm{t}, J=7.2 \mathrm{~Hz}, 2 \mathrm{H}, \mathrm{CH}_{2} \mathrm{~N}\right), 3.16-2.40(\mathrm{br} \mathrm{m}, 10 \mathrm{H}$ ), 2.12-1.50 (br m, 21H), 1.46-1.07 (br m, 30H), 1.14 (br t, $J=7.4 \mathrm{~Hz}, 6 \mathrm{H}, \mathrm{CH}_{2} \mathrm{CH}_{3}$ ) 0.95 (t, $J=7.4 \mathrm{~Hz}$, $\left.3 \mathrm{H}, \mathrm{CH}_{2} \mathrm{CH}_{3}\right)$.

${ }^{13} \mathrm{C}$ NMR $\left(\mathrm{CD}_{2} \mathrm{Cl}_{2}\right): \square=176.2,174.2,169.3,165.9,158.4,152.6,152.4,151.5,150.8,147.3,141.5$, 139.7, 134.2, 131.9, 131.6, 131.0, 126.7, 110.9, 110.0, 96.8, 69.0, 68.6, 64.9, 52.0, 50.8, 50.1, 48.7, $48.3,42.6,41.6,36.8,35.2,35.1,32.2,31.6,30.5,30.2-29.8,29.7,29.5,29.3,26.5,26.4,20.2,14.0$, $12.5,9.8$.

FTIR $(\mathrm{NaCl}): \mathrm{cm}^{-1}=3269,3215,3162,3058,2929,2856,1683,1631,1580,1547,1439,1435,1356$, 1317, 1281, 1216, 1164, 1060, 968, 933, 895, 847, 804, 751, 692, 682.

$\operatorname{UVVIS}\left(\mathrm{CH}_{2} \mathrm{Cl}_{2}\right): \square \max (\mathrm{nm})=271(\mathrm{sh}), 315(\mathrm{sh})$. 
Multi-Step Self-Assembly: Starting with Hydrogen-Bonding(4d-6d). Copolymers 4c-6c were dissolved in $\mathrm{CD}_{2} \mathrm{Cl}_{2}(0.7 \mathrm{~mL}$ per $50 \mathrm{mg})$. To these solutions, one stoichiometric equivalent (based on the number of pincer complexes) of a pyridine stock solution (in $\mathrm{CD}_{2} \mathrm{Cl}_{2}$ ) was added. Subsequently, one equivalent of $\mathrm{AgBF}_{4}$ (stock solution in nitromethane) was introduced to the mixture and the color changed from dark to pale green. Upon complete coordination, crude polymers $\mathbf{4 d - 6 d}$ were obtained by filtration over Celite $\AA$, removal of the solvent, and drying on high vacuum. To remove excess $\mathrm{AgCl}$ salts, the polymers were subjected to repeated (3X) redissolution in $\mathrm{CH}_{2} \mathrm{Cl}_{2}$ followed by filtration over Celite $₫$, removal of the solvent, and extensive drying on high vacuum to provide pure $\mathbf{4 d - 6} \mathbf{d}$.

${ }^{1} \mathrm{H}$ NMR $\left(\mathrm{CD}_{2} \mathrm{Cl}_{2}\right): \square=10.53(\mathrm{br} \mathrm{s}, 1 \mathrm{H}, \mathrm{NH}), 9.66$ (br s, 2H, CONH), $8.04\left(\mathrm{~s}, 2 \mathrm{H}, \mathrm{Pyr}_{\square} \mathrm{H}\right), 7.76(\mathrm{~m}, 1 \mathrm{H}$, $\left.\operatorname{Pyr}_{\square} \mathrm{H}\right), 7.63\left(\mathrm{~m}, 6 \mathrm{H}, \mathrm{SPh} \& \mathrm{Pyr}_{\square}\right), 7.49-7.37$ (br m, 6H, SPh), $7.27\left(\mathrm{~m}, 2 \mathrm{H}, \mathrm{Pyr}_{\square}\right), 7.06(\mathrm{~m}, 1 \mathrm{H}$, $\left.\mathrm{CH}=\mathrm{CCH}_{3}\right), 6.68(\mathrm{~s}, 2 \mathrm{H}, \mathrm{ArH}), 5.42-5.17$ (br m, 4H, CH=CH), 4.74 (br s, 4H, $\left.\mathrm{CH}_{2} \mathrm{~S}\right), 4.07-3.94$ (br m, $6 \mathrm{H}, \mathrm{CH}_{2} \mathrm{O}$ ), 3.84 (br t, J = 6.4 Hz, 2H, CH $\mathrm{CH}_{2} \mathrm{O}$ ), 3.70 (t, J=7.2 Hz, 2H, $\mathrm{CH}_{2} \mathrm{~N}$ ), 3.16-2.40 (br m, 10H), 2.12-1.50 (br m, 21H), 1.46-1.07 (br m, 30H), 1.14 (br t, $\left.J=7.4 \mathrm{~Hz}, 6 \mathrm{H}, \mathrm{CH}_{2} \mathrm{CH}_{3}\right) 0.95(\mathrm{t}, J=7.4 \mathrm{~Hz}$, $3 \mathrm{H}, \mathrm{CH}_{2} \mathrm{CH}_{3}$ ).

${ }^{13} \mathrm{C}$ NMR $\left(\mathrm{CD}_{2} \mathrm{Cl}_{2}\right): \square=176.2,174.2,169.3,165.9,158.4,152.6,152.4,151.5,150.8,147.3,141.5$, $139.7,134.2,131.9,131.6,131.0,126.7,110.9,110.0,96.8,69.0,68.6,64.9,52.0,50.8,50.1,48.7$, 48.3 , 42.6, 41.6, 36.8, 35.2, 35.1, 32.2, 31.6, 30.5, 30.2-29.8, 29.7, 29.5, 29.3, 26.5, 26.4, 20.2, 14.0, $12.5,9.8$.

FTIR $(\mathrm{NaCl}): \mathrm{cm}^{-1}=3269,3215,3162,3058,2929,2856,1683,1631,1580,1547,1439,1435,1356$, $1317,1281,1216,1164,1060,968,933,895,847,804,751,692,682$.

UVVIS $\left(\mathrm{CH}_{2} \mathrm{Cl}_{2}\right): \square \max (\mathrm{nm})=271(\mathrm{sh}), 315(\mathrm{sh})$.

One-Step Orthogonal Self-Assembly (4d- 6d). Copolymers 4a-6a were dissolved in $\mathrm{CD}_{2} \mathrm{Cl}_{2}(0.7 \mathrm{~mL}$ per $50 \mathrm{mg}$ ). To these solutions, a stock solution comprised of 1.5 stoichiometric equivalents of $\mathrm{N}$ - 
butylthymine (based on the number of diaminopyridine moieties), 1 equivalent of pyridine, and 1

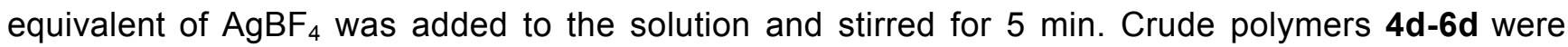
obtained by filtration over Celite ${ }^{\circledR}$, removal of the solvent, and drying on high vacuum. To remove excess $\mathrm{AgCl}$ salts, the polymers were subjected to repeated (3X) redissolution in $\mathrm{CH}_{2} \mathrm{Cl}_{2}$ followed by filtration over Celite ${ }^{\circledR}$, removal of the solvent, and extensive drying on high vacuum to provide pure 4d-6d.

${ }^{1} \mathrm{H}$ NMR $\left(\mathrm{CD}_{2} \mathrm{Cl}_{2}\right): \square=10.53(\mathrm{br} \mathrm{s}, 1 \mathrm{H}, \mathrm{NH}), 9.66$ (br s, 2H, CONH), $8.04\left(\mathrm{~s}, 2 \mathrm{H}, \mathrm{Pyr}_{\square} \mathrm{H}\right), 7.76(\mathrm{~m}, 1 \mathrm{H}$, $\left.\operatorname{Pyr}_{\square} H\right), 7.63\left(\mathrm{~m}, 6 \mathrm{H}, \mathrm{SPh} \& \mathrm{Pyr}_{\square}\right), 7.49-7.37(\mathrm{br} \mathrm{m}, 6 \mathrm{H}, \mathrm{SPh}), 7.27\left(\mathrm{~m}, 2 \mathrm{H}, \mathrm{Pyr}_{\square}\right), 7.06(\mathrm{~m}, 1 \mathrm{H}$, $\mathrm{CH}=\mathrm{CCH}_{3}$ ), 6.68 (s, 2H, ArH), 5.42- 5.17 (br m, 4H, CH=CH), 4.74 (br s, 4H, $\mathrm{CH}_{2} \mathrm{~S}$ ), 4.07-3.94 (br m, $6 \mathrm{H}, \mathrm{CH}_{2} \mathrm{O}$ ), 3.84 (br t, J = 6.4 Hz, 2H, $\mathrm{CH}_{2} \mathrm{O}$ ), 3.70 (t, J = 7.2 Hz, 2H, $\left.\mathrm{CH}_{2} \mathrm{~N}\right), 3.16-2.40(\mathrm{br} m, 10 \mathrm{H})$, 2.12-1.50 (br m, 21H), 1.46-1.07 (br m, 30H), 1.14 (br t, J = 7.4 Hz, 6H, $\left.\mathrm{CH}_{2} \mathrm{CH}_{3}\right) 0.95$ (t, J = 7.4 Hz, $\left.3 \mathrm{H}, \mathrm{CH}_{2} \mathrm{CH}_{3}\right)$.

${ }^{13} \mathrm{C}$ NMR $\left(\mathrm{CD}_{2} \mathrm{Cl}_{2}\right): \square=176.2,174.2,169.3,165.9,158.4,152.6,152.4,151.5,150.8,147.3,141.5$, $139.7,134.2,131.9,131.6,131.0,126.7,110.9,110.0,96.8,69.0,68.6,64.9,52.0,50.8,50.1,48.7$, $48.3,42.6,41.6,36.8,35.2,35.1,32.2,31.6,30.5,30.2-29.8,29.7,29.5,29.3,26.5,26.4,20.2,14.0$, $12.5,9.8$.

FTIR (NaCl): $\mathrm{cm}^{-1}=3269,3215,3162,3058,2929,2856,1683,1631,1580,1547,1439,1435,1356$, $1317,1281,1216,1164,1060,968,933,895,847,804,751,692,682$.

UVVIS $\left(\mathrm{CH}_{2} \mathrm{Cl}_{2}\right)$ : $\square \max (\mathrm{nm})=271(\mathrm{sh}), 315(\mathrm{sh})$.

Determination of Association Constants. Association constants were measured by titration of a $0.010 \mathrm{M}$ solution of polymers $\mathbf{7 a}, \mathbf{5 a}$, and $\mathbf{5 b}$ with a $0.005 \mathrm{M}$ solution of $\mathrm{N}$-butylthymine, and monitoring of the chemical shift of the amide protons. The molarity of the polymer solutions are based 
on the number of recognition units. The computer program ChemEquili was used for evaluation of the data $^{2}$.

${ }^{1}$ Pollino, J. M.; Stubbs, L. P.; Weck; M. Macromolecules, 2003, 36, 2230-2234.

${ }^{2}$ ChemEquili 6.1, V. Solov'ev 1996-1998. 